\title{
Assessment of drought tolerant cotton genotypes based on seedling \& physiological attributes at different moisture levels
}

\author{
Muhammad Akbar ${ }^{1 *}$ and Sayed Bilal Hussain ${ }^{2}$ \\ 1. Bahuddin Zakaryia University, Multan-Pakistan \\ 2. Institute of Molecular Biology \& Biotechnology, BahuddinZakaryia University, Multan-Pakistan \\ *Corresponding author's email: malikakbarccri@gmail.com
}

Citation

Muhammad Akbar and Sayed Bilal Hussain. Assessment of drought tolerant cotton genotypes based on seedling \& physiological attributes at different moisture levels. Pure and Applied Biology. Vol. 8, Issue 1, pp93-107. http://dx.doi.org/10.19045/bspab.2018.700167

\begin{tabular}{llll}
\hline \hline Received: 07/07/2018 & Revised: 28/09/2018 & Accepted: 04/09/2018 & Online First: 12/10/2018 \\
\hline \hline
\end{tabular}

\section{Abstract}

The potential of any crop variety is subjected to various environmental conditions and a large fraction of the potential yield cannot be attained. For this study, fifty cotton genotypes of different origin were collected from gene pool of Central Cotton Research Institute, Multan and screened in the glass house. These genotypes were also evaluated under the field condition to obtain parents that possess better tolerance against drought. Initial screening of these genotypes was conducted in the glass house under three moisture levels 100, 70 and $40 \%$ at field water capacity. Data were recorded for root length, fresh root weight, dry root weight, root shoot length ratio, shoot length; fresh shoot weight, dry shoot weight, fresh root shoot weight ratio and dry root shoot weight ratio in glass house. Five drought tolerant genotypes (BH-176, MPS11, DPL-45, Tree cotton and Cyto-62) and three susceptible genotypes (GS-444, Cooker-315, MS-64) were identified. Highly significant differences found among genotypes for all seedling characters. Further evaluation was done in field on the basis of various physiological parameters i.e. relative water content, excised leaf water loss, cell injury, leaf water potential, osmotic potential, net photosynthesis rate, transpiration rate and stomatal conductance under normal and water stress conditions. Same genotypes were confirmed under field studies. Highly significant genotypic variation existed among genotypes for all the physiological traits. The predicting genotypes may be selected and exploited to improve drought tolerance for the cotton crop.

Keywords: Cotton; Drought; Physiological traits; Pakistan; Seedling traits; Tolerance

\section{Introduction}

Cotton being a miraculous fibre that has a variety of uses, from fashion to home stuff to medical products. Cotton is noted for its versatility, appearance and performance. Cotton moved from field to fabrics as it provides thousands of useful products and supports millions of jobs. It is the key source of lint along with its by-products i.e. cotton seed oil, cake, hulls, and linters. In the international cotton set-up, Pakistan has the peculiarity in terms of cotton production and area. Cotton is the second most significant crop of our country after wheat, in terms of area, production and value added. Pakistan is the fifth largest producer of cotton all over the globe by producing 2170,000 million tons, the third leading exporter of raw cotton, the 
fourth major consumer of cotton $(2467,000$ million tons), and the prime exporter of cotton yarn [1].

The potential of any crop variety is subjected to various environmental conditions and a large fraction of the potential yield cannot be attained. It is speculated that crop attains less than $25 \%$ of the potential yield due to the adverse environmental conditions, and low water availability affected crop productivity nearly as much as all the other environmental factors combined $[2,3]$. Amongst the abiotic stresses, deficiency of soil water constitutes a primary limitation to crop productivity in many regions of the world $[4,5]$. Water deficit is not only the cause of differences between actual yield and the potential yield, but it also results in yield instability of crops. Both surface and ground water resources in Pakistan are inadequate to meet the growing requirements for irrigation of agricultural crops. Drought has a very critical effect on growth, yield and quality characters [6]. Limited water supply and high-energy costs can also decrease the yield of irrigated cotton. $[7,8]$ have focused on root systems to enlarge water use efficiency. Root characteristics can be significant in predicting the response of plants to drought. Drought effect not only decreases plant height, shoot growth rate, and yield but also diminishes root growth. [9] have exhibited that root growth is less sensitive than shoot growth. Development of such genotypes which appears to be practicable, cost-effective and to tackle the problem of water shortage is an alternate way by genetic variation.

It has been found from earlier studies that varieties/cultivars in each species vary from one another in their actions under drought conditions, signifying that drought tolerance in these groups can be improved through breeding. Physiological traits linked with drought tolerance in cotton have strong relationship with yield parameters. For example, photosynthetic rate; which significantly decreases with the imposition of water stress, can be effectively used for germplasm screening under drought conditions $[10,11]$. Since, the response of germplasm with genetic variability may exhibit differential response under normal and water deficit conditions, regular screening of emerging germplasm need to be carried out for better adaptability and sustainable production.

This study will help to understand the relationship of different seedling and physiological traits of cotton and their direct and indirect effects. The following studies were, therefore, to evaluate advanced cotton genotypes for drought tolerance characteristics under field conditions.

\section{Materials and methods}

\section{Screening of cotton genotypes in glass} house

Initial screening was conducted in glasshouse under three moisture levels $100 \%, 70 \%$ and $40 \%$ at field water capacity. Seeds of all 50accessions were collected from the available stock at Central Cotton Research Institute, Multan. Seeds of accessions were planted during November, 2012 in polythene bags. Four hundred and fifty polythene bags with three repeats using completely randomized design were divided into three sets. One set was treated as control $\left(\mathrm{T}_{0}\right) 100 \%$ and the other two as water stressed conditions $70 \%\left(\mathrm{~T}_{1}\right)$ and $40 \%$ field capacity $\left(\mathrm{T}_{2}\right)$.

Seedlings grown under control condition with $100 \%$ water level $\left(\mathrm{T}_{0}\right)$ were watered daily to keep the soil at field capacity. Water stress condition was developed by withholding water supply to the seedlings grown under water stress condition at two water level of $70 \%\left(\mathrm{~T}_{1}\right) \& 40 \%\left(\mathrm{~T}_{2}\right)$, and the effect of water stress was monitored visually and with soil moisture meter (HH2 Theta Probe Type, Delta-T device, Cambridge, England).

The data for each accession were recorded after 45 days of emergence. Plants were 
removed from the pots and gently washed to remove all sand. The plants were spread on paper for the measurement of fresh root length $(\mathrm{g})$ and shoot length $(\mathrm{cm})$. Plants were cut into roots and shoots, and fresh root weight (g) and fresh shoot weight (g) were calculated. Then roots and shoots dried for $72 \mathrm{~h}$ at $60^{\circ} \mathrm{C}$. Genotypes were evaluated for root length, fresh root weight, dry root weight, root shoot length ratio, shoot length, fresh shoot weight, dry shoot weight, fresh root weight, fresh shoot weight ratio and dry root shoot weight ratio.

\section{Screening of cotton genotypes in field condition}

Fifty cotton genotypes were screened under two irrigation levels i.e., well watered $\left(\mathrm{T}_{0}\right)$ and water-limited $\left(\mathrm{T}_{1}\right)$ in the field. The experimental design was Randomized Complete Block (RCBD) with three replications. Seeds of the genotypes were planted by maintaining $75 \times 30 \mathrm{~cm}$ row to row and plant to plant distance respectively. In order to avoid the rainfall effect, soil between the rows was covered with polythene sheet during the rainy period i.e. July-August as done by [12]. Data of relative water content, excised leaf water loss, cell injury, leaf water potential, osmotic potential, net photosynthesis rate, transpiration rate and stomatal conductance were recorded.

\section{Statistical analysis}

The average data of all the seedling traits were subjected to statistical analysis. The least significance difference (LSD) was calculated at probability of $0.05 \%$. Software packages of SPSS version 19 was used for data analysis [13].

\section{Results and discussion}

\section{Response of cotton genotypes in glass house}

Variations were significant among all parameters for 50 genotypes (Table 1) under both stress levels suggested that root and shoot parameters as reliable traits for screening cotton germplasm for moisture stress. Genetic variation in 50 genotypes under drought and controlled conditions suggested the importance of root and shoot parameters in breeding programs $[14,15]$. Highly significant differences were also noted in respect of genotypes $(\mathrm{G})$, replication (R) as well as interaction of genotypes with replications $(\mathrm{G} \times \mathrm{R})$. Stress produced a range of responses on the seedling traits measured. The presence of variability among genotypes for different traits under water stressed conditions has been reported $[16,17]$. It is evident from the findings that the mean squares between all the accessions for all the seedling traits were highly significant under normal and moisture stress conditions $(\mathrm{P} \leq$ $0.01)$.

\section{Root parameters}

Results exhibited from (Table 2) that mean for all the root parameters under controlled were ranged from $6.77 \mathrm{~cm}$ for (MS-64) to $13.97 \mathrm{~cm}$ for DPL-45. On the average basis DPL-45 had maximum root length $(11.56 \mathrm{~cm})$ followed by BH-176 $(11.42 \mathrm{~cm})$, Tree cotton $(11.33 \mathrm{~cm})$ and MPS-11 $(10.72 \mathrm{~cm})$. These genotypes performed better in all the conditions and these are considered as drought tolerant genotypes while entries GS444 showed minimum root length following MS-64, Coker-315 and CIM-84 with root length of $4.8,4.99, \quad 5.4$ and $5.6 \mathrm{~cm}$ respectively. Due to drastic reduction in root length in stressed condition, these accessions may be rated as susceptible to water stress. [18] reported in his findings that in cotton, root length was increased in mild and initialstage drought stress, but long-time reduced the root length as compared to control plants. On overall mean performance for fresh root weight, Tree cotton, F-14, GH-11-9-75 and MPS-11 had maximum mean values of $0.394,0.387,0.380$ and $0.374 \mathrm{~g}$ respectively while minimum fresh root weight was predicted by VS-212 (0.222g), Cooker-315 (0.224g), CIM-608 (0.226g) and GS-444 $(0.230 \mathrm{~g})$. Root length-shoot length ratio 
under controlled and stressed conditions was maximum in F-14 followed by DPL-45, Cyto-62 and BH-176 with 0.910, 0.810, 0.794 and 0.778 respectively. These findings were very similar to [19]. [20] Reported that accessions may be rated as susceptible due to reduction in root lengths in stressed condition. Minimum ratio was exhibited by CRIS-533 (0.404), Stoneville-603 (0.436), CIM-84 (0.444) and VS-212 (0.457). [21, 22] also reported that root related parameters in cotton are directly associated with drought tolerance.

\section{Shoot parameters}

For drought tolerance, shoot length has been used as a selection parameter $[23,24]$. Under $100 \%$ moisture levels (Table 3) shoot length ranged from $12.07 \mathrm{~cm}$ (MS-64) to $19.67 \mathrm{~cm}$ (VS-212) similarly at $70 \%$ and $40 \%$ moisture levels its range was 9.6-15.8 and 4.07$11.50 \mathrm{~cm}$ respectively. Data on mean performance of shoot length exhibited that maximum shoot length was found in MPS11, Tree cotton, BH-176 and VS-212, with values of $15.0,14.9$ and $14.9 \mathrm{~cm}$ respectively and these entries were less affected by moisture stress revealing that these are tolerant as compared to remaining entries. Minimum shoot length was shown by MS-64 $(7.87 \mathrm{~cm}), \mathrm{GS}-444(8.0 \mathrm{~cm}), \mathrm{F}-14 \quad(9.29 \mathrm{~cm})$ and Cooker-315 $(9.66 \mathrm{~cm})$. [25] Exhibited that shoot length can be used as selection criteria for drought tolerance.

On overall performance under normal and stress conditions, shoot weight mean values showed that Cyto-62 and $\mathrm{BH}-176$ were the most tolerant entries showing better performance with maximum shoot weight of 1.91 and $1.82 \mathrm{~g}$ respectively. The genotypes GS-444, L-229-29-71and Cooker-315 had minimum fresh shoot weight of $0.93,0.97$ and $1.03 \mathrm{~g}$ respectively.

In cotton, shoot dry weight is commonly used as a parameter for screening cotton genotypes against drought stress. Under normal condition; minimum dry shoot weight was exhibited by GS-444, Coker-315 and MS-64 with an average value of $0.28,0.27$ and $0.27 \mathrm{~g}$ respectively. Minimum values for shoot weight at both stress levels were shown by the genotypes Cooker-315(0.19), MS64(0.189) and GS-444(0.151g) respectively at $70 \%$ moisture level. While under $40 \%$ moisture level it was found as $0.109,0.107$, $0.103 \mathrm{~g}$ and at mean of three levels it was observed as $0.192,0.189,0.178 \mathrm{~g}$ respectively (Table 3).

Based on root and shoot weight ratio; all accessions again appeared to respond differently both under normal and stressed conditions. On average based performance, under controlled and stress conditions maximum ratio was exhibited by the genotype L-229-29-71 followed by CRIS-9, F-14 and BP-52 with mean ratio of 0.91, $0.338,0.327,0.297$ and 0.296 respectively. Minimum ratio was exhibited by NIAB-112, VS-212, CIM-84 and CIM-608 with values of $0.1550 .139,0.145,0.151$ respectively.

Entries performed better under stress condition exhibited low dry root dry shoot weight ratio as the stress level increased. Under $40 \%$ moisture level; entries i.e. Cooker-315 (0.322) and MS-64 (0.300) with poor performance under maximum stress showed maximum ratio while minimum ratio was observed for BH-176 followed by Cyto62, DPL-45, MPS-11 and Tree cotton with ratios of $0.123,0.132,0.133,0.134$ and 0.141 respectively. It was concluded that diminution in performance for root and shoot parameters is due to the shortage of water supply and was also reported in the study of $[20,26]$.

\section{Response of cotton genotypes under field condition}

Drought stress causes a wide range of adverse effects on physiological traits as well as productivity of cotton crop. Under drought conditions specifically in cotton leaves, net photosynthetic rate, transpiration rate, stomatal conductance, carboxylation 
efficiency and water potential decreased significantly [27].

The data obtained for all physiological traits under normal and stress conditions were subjected to pooled analysis of variance. Analysis of variance showed highly significant differences $(\mathrm{P} \leq 0.01)$ among cotton genotypes (Table 4). Genotype $\mathrm{x}$ environment interaction was also found highly significant for all the traits.

\section{Relative water content (RWC).}

In cotton plants reduction of water loss through leaves is an important phenomenon under drought stress. Wilting and rolling of leaves result in water loss [28] Relative Water Content was reduced remarkably under stress condition. Maximum RWC was found in CIM-591 followed by BH-176, MPS-11, Cyto-62 and DPL-45 with average values of $83.00,81.00,80.67,80.33$ and 80.33 respectively under controlled condition (Table 5). While, minimum RWC was exhibited by MS-64 (44.0), Cooker-315 (45.3) and GS-444 (63.3). Maximum value was shown by MPS-11 (59.33), BH-176 (58.67), Karishma (54.67) and DPL-45 (54.33) while entries with lowest values of relative water content were GS-444, CRIS510 and Cooker-315 with average value of $32.33,34.67$ and 40.08 respectively in drought. On basis of grand mean obtained from normal and drought condition RWC was 42.33 to 70.00 . Our results were very similar to $[29,30]$ who reported that relative water content and water potential reduction occur when leaves are subjected to drought condition

\section{Excised leaf water loss (ELWL).}

Excised leaf water loss had been widely used in various crops plants as a selection parameter under drought condition [31, 32]. Minimum excised leaf water loss was exhibited by GS-444 (0.53) and B-452, Cooker-315 (0.57). Maximum value was found in BH-176 (1.47), Cyto-62, Tree cotton, MPS-11 (1.20), and Stoneville (1.10) while entries with lowest values of excised leaf water loss were MS-64, Cooker-315, Cooker-312, and GS-444 with average value of $0.23,0.33,0.37$, and 0.43 respectively under drought condition. While at controlled condition, maximum excised leaf water loss was observed in BH-176 followed by Cyto62, Tree cotton, MPS-11, and DPL-45 with average value of $2.03,2.00,1.97,1.93$ and 1.87 respectively. On the basis of grand mean obtained from normal and drought conditions, excised leaf water loss range was 0.43-1.75 (Table 5).

\section{Cell injury}

Relative cell injury has been considered as a reliable parameter for screening against abiotic stress tolerance [33, 34]. Cell injury was also reduced remarkably in stress condition. Minimum cell injury was exhibited by BH-176, DPL-45 Tree cotton, CIM-608 and Cyto-62 with an average values of $21.1,21.47,22.03,22.07$ and 22.27 respectively. Under drought condition; maximum value was shown by MS-64 (67.10), GS-444 (66.07), and Cooker$315(65.5)$ while entries with lowest values of cell injury were Tree cotton (31.60), Cyto62(36.57). DPL-45 (36.63), MPS-11 (38.07) and $\mathrm{BH}-176$ (39.30). Under controlled condition, maximum cell injury was shown by MS-64 followed by Sun-02, and GS-444 with an average values of 42.57, 38.87 and 38.37 respectively (Table 5). On the basis of grand mean obtained from normal and conditions cell injury range was 26.82 to 54.83 .

\section{Leaf water potential}

Under controlled condition, maximum leaf water potential was found in Tree cotton (17.00), Stoneville-603(-17.00), BH-176 (20), DNH-105 (-20.33), Cyto-62(-18.00) and GS-444 with value of (-21.00) (Table 5).Similarly, minimum leaf water potential was exhibited by Cooker-315 (-29.33), MS64 (-27.33) and GS-444, (-27.00). Under drought condition, maximum value was 
shown by Tree cotton (-21.00), BH-176(22.00), Cyto-62 (-22.33), Stoneville-603 (22.33) and MPS-11 (-22.67).While Cooker315, MS-64, NIAB-78 and GS-444 had lowest values of leaf water potential (-31.67), (-30.33), (-30.33), (-31.00). On the basis of grand mean, leaf water potential range was $30.5-20.50$.

\section{Osmotic potential}

Under drought condition, negative effects have been observed on osmotic balance therefore, plants tend to accumulate different organic and inorganic molecules to reduce the osmotic potential [29]. Osmotic potential was also markedly reduced in stress condition. Under controlled condition; maximum value was shown by $\mathrm{BH}-176(-$ $0.141)$, DPL-45 (-0.141), Tree cotton (0.144), MPS-11 (-0.145) and Cyto-62($0.153)$, while entries with lowest values of osmotic potential included PB-38 (-0.270), MS-64(-0.269) and IUB-2011(-0.269) (Table 6). Under drought condition, maximum osmotic potential was shown by $\mathrm{BH}-176$ followed by Cyto-62, MPS-11, DPL-45 and BP-52 with average value of $-0.188,-0.242$,$0.254,-0.254$, and -0.256 respectively. Similarly, minimum osmotic potential was exhibited by cooker-315 (-0.380), MS-64($0.365)$ and CIM-43 (-0.355). On the basis of grand mean obtained from normal and drought condition osmotic potential range was -0.324 to -0.165 .

\section{Gas exchange parameters}

Net photosynthetic rate $\left(\mathrm{P}_{\mathrm{N}}\right)$, stomatal conductance $\left(\mathrm{g}_{\mathrm{s}}\right)$ and transpiration rate $(\mathrm{E})$ of 50 cotton genotypes were measured under normal (Table 6) and stress conditions (Table 6). Water deficit induced substantial reduction in $\left(\mathrm{P}_{\mathrm{N}}\right)$ compared to the normal condition [35]. Reduction in $\mathrm{P}_{\mathrm{N}}$ was apparent in genotypes GS-44 (11.10 m mol CO $2 \mathrm{~m}^{-2} \mathrm{~s}^{-}$ $\left.{ }^{1}\right)$ and Cooker-315 (11.13 $\left.\mathrm{m} \mathrm{mol} \mathrm{CO}_{2} \mathrm{~m}^{-2} \mathrm{~s}^{-1}\right)$ under drought. However, water deficit caused less reduction for $\mathrm{P}_{\mathrm{N}}$ in DPL-45, MPS-11, Cyto-62 and Tree cotton with average value of $13.02,13.00,12.96$ and $12.94 \mathrm{~m} \mathrm{~mol} \mathrm{CO}_{2}$ $\mathrm{m}^{-2} \mathrm{~s}^{-1}$ respectively. On the basis of grand mean obtained from normal and drought conditions, $\mathrm{P}_{\mathrm{N}}$ range was 13.08-14.9 $\mu \mathrm{mol} / \mathrm{m}^{2} / \mathrm{s}$. Photosynthesis was affected under drought condition which is predicted in findings of $[36,37]$. These studies revealed that under drought condition photo synthesis was reduced in cotton which in turn affects growth and yield.

Ninety per cent of water losses (transpiration) from plants occur though stomata openings under drought; stomatal conductance $\left(\mathrm{g}_{\mathrm{s}}\right)$ was maximum in the entries (MPS-11 (25.61 $\left.\mathrm{mmol} \mathrm{CO}_{2} \mathrm{~m}^{-2} \mathrm{~s}^{-1}\right)$, BH-176 (25.52 $\mathrm{mmol} \mathrm{CO}_{2}$ $\left.\mathrm{m}^{-2} \mathrm{~s}^{-1}\right)$, DPL-45 (25.05mmol CO $\left.\mathrm{Cm}^{-2} \mathrm{~s}^{-1}\right)$ and Tree cotton (25.36 mmol $\left.\mathrm{CO}_{2} \quad \mathrm{~m}^{-2} \mathrm{~s}^{-1}\right)$ revealing that under stress condition these entries were performing better while minimum $\mathrm{g}_{\mathrm{s}}$ was exhibited by GS-444 and MS-64 with $\mathrm{g}_{\mathrm{s}}$ of $21.63 \mathrm{mmol} \mathrm{CO}_{2} \mathrm{~m}^{-2} \mathrm{~s}^{-1}$ and $21.69 \mu \mathrm{mol} / \mathrm{m}_{2} \mathrm{~s}$ revealing that these were badly affected by stress [29]. The genotypes BH-176, Cyto-62, MPS-11, Tree cotton and DPL-45 had the highest stomatal conductance where as $\mathrm{g}_{\mathrm{s}}$ was lowest in CIM84 (30.61 mmol CO $\left.\mathrm{m}^{-2} \mathrm{~s}^{-1}\right)$, MS-64 (31.28 $\left.\mathrm{mmol} \mathrm{CO}_{2} \mathrm{~m}^{-2} \mathrm{~s}^{-1}\right)$, GS-444 (31.45 mmol CO 2 $\left.\mathrm{m}^{-2} \mathrm{~s}^{-1}\right)$ and cooker-315 (32.07 $\mathrm{mmol} \mathrm{CO}_{2} \mathrm{~m}^{-}$ ${ }^{2} \mathrm{~s}^{-1}$ ) in controlled condition.

Drought stress caused drastic reduction in transpiration rate, genotypes with an average decline of $45 \%$. Again, genotype $\mathrm{BH}-176$, MPS-11, DPL-45 and Tree cotton also showed higher values for $\mathrm{E}$ under controlled condition while entries GS-444, MS-64 and Cooker-315 exhibited minimum E under normal condition. When stress was applied, same entries DPL-45, BH-176, Tree cotton, MPS-11 and Cyto-62 performed better in spite of stress revealing their tolerance to drought while entries with minimum value were MS-64, Cooker-315 and GS-444 revealing their susceptible behavior for drought. 
Table 1. Mean square for seedling traits of 50 Gossypium hirsutum $\mathbf{L}$. accessions measured under control and water stressed conditions

\begin{tabular}{|c|c|c|c|c|c|c|c|c|c|c|}
\hline SOV & DF & RL & FRW & DRW & SL & SW & DSW & RL:SL & FRW:FSW & DRW:DSW \\
\hline REP & 2 & 867.602 & 1.22450 & 0.01156 & 2743.20 & 29.1753 & 1.81619 & 0.094410 & 0.031554 & 0.1434173 \\
\hline GENO & 49 & 21.953 & 0.01856 & $1.215 \mathrm{E}-04$ & 26.79 & 0.4460 & 0.02758 & 0.106597 & 0.017796 & 0.0048114 \\
\hline ERROR & 300 & 0.106 & 0.00033 & $2.142 \mathrm{E}-06$ & 0.20 & 0.0040 & 0.00066 & 0.001370 & 0.000321 & 0.0003783 \\
\hline
\end{tabular}

SOV, Source of variation; Df, degree of freedom; SL, shoot length; RL, root length; SL/RL ratio, ratio of shoot to root length; RW, root weight; SW, shoot weight; SW/RW ratio, ratio between shoot and root weight; DRW/DSW ratio, dry root weight dry shoot weight ratio

Table 2. Mean values of 50 Gossypium hirsutum L. accessions for Root parameters grown in control and water stress conditions

\begin{tabular}{|c|c|c|c|c|c|c|c|c|c|c|c|c|}
\hline \multirow[b]{2}{*}{ Genotypes } & \multicolumn{4}{|c|}{$\mathbf{R L}$} & \multicolumn{4}{|c|}{ FRW } & \multicolumn{4}{|c|}{ Root Shoot Length ratio } \\
\hline & 100 & 70 & 40 & $\begin{array}{c}\text { M. } \\
\text { Value }\end{array}$ & 100 & 70 & 40 & $\begin{array}{c}\text { M. } \\
\text { Value }\end{array}$ & 100 & 70 & 40 & $\begin{array}{c}\text { M. } \\
\text { Value }\end{array}$ \\
\hline GS-444 & 7.30 & 4.40 & 2.80 & 4.83 & 0.333 & 0.203 & 0.155 & 0.230 & 0.591 & 0.589 & 0.675 & 0.618 \\
\hline Cyto-124 & 8.43 & 6.20 & 4.93 & 6.52 & 0.386 & 0.275 & 0.241 & 0.301 & 0.548 & 0.511 & 0.617 & 0.558 \\
\hline DNH-105 & 9.00 & 6.43 & 4.13 & 6.52 & 0.377 & 0.285 & 0.241 & 0.301 & 0.518 & 0.481 & 0.399 & 0.466 \\
\hline CRIS-533 & 7.87 & 5.47 & 4.07 & 5.8 & 0.373 & 0.262 & 0.213 & 0.283 & 0.432 & 0.381 & 0.399 & 0.404 \\
\hline MPS-27 & 8.70 & 6.53 & 5.17 & 6.8 & 0.389 & 0.287 & 0.208 & 0.295 & 0.575 & 0.567 & 0.587 & 0.576 \\
\hline CIM-506 & 8.53 & 5.80 & 4.70 & 6.34 & 0.347 & 0.275 & 0.215 & 0.279 & 0.586 & 0.537 & 0.553 & 0.559 \\
\hline TH-112/05 & 9.43 & 6.47 & 3.87 & 6.59 & 0.401 & 0.311 & 0.219 & 0.310 & 0.536 & 0.510 & 0.455 & 0.500 \\
\hline PB-896 & 10.50 & 7.40 & 4.73 & 7.54 & 0.409 & 0.301 & 0.266 & 0.325 & 0.575 & 0.600 & 0.585 & 0.586 \\
\hline Sun-02 & 10.13 & 7.20 & 3.93 & 7.09 & 0.425 & 0.319 & 0.268 & 0.338 & 0.519 & 0.492 & 0.409 & 0.474 \\
\hline CIM-573 & 11.47 & 8.17 & 5.40 & 8.34 & 0.452 & 0.295 & 0.175 & 0.307 & 0.666 & 0.646 & 0.724 & 0.679 \\
\hline BH-176 & 13.70 & 11.63 & 8.93 & 11.42 & 0.465 & 0.320 & 0.271 & 0.352 & 0.731 & 0.742 & 0.860 & 0.778 \\
\hline CIM-591 & 9.40 & 6.50 & 4.37 & 6.76 & 0.315 & 0.222 & 0.205 & 0.247 & 0.556 & 0.551 & 0.555 & 0.554 \\
\hline NIA-80 & 10.30 & 6.80 & 4.80 & 7.3 & 0.359 & 0.283 & 0.242 & 0.295 & 0.634 & 0.665 & 0.735 & 0.678 \\
\hline CRIS-510 & 9.50 & 5.97 & 4.23 & 6.57 & 0.345 & 0.260 & 0.208 & 0.271 & 0.576 & 0.487 & 0.510 & 0.524 \\
\hline VH-300 & 8.90 & 6.80 & 5.00 & 6.9 & 0.318 & 0.245 & 0.214 & 0.259 & 0.476 & 0.457 & 0.531 & 0.488 \\
\hline VS-212 & 8.63 & 6.20 & 5.10 & 6.64 & 0.279 & 0.219 & 0.169 & 0.222 & 0.439 & 0.407 & 0.524 & 0.457 \\
\hline
\end{tabular}


Akbar \& Hussain

\begin{tabular}{|c|c|c|c|c|c|c|c|c|c|c|c|c|}
\hline MPS-11 & 12.87 & 10.70 & 8.60 & 10.72 & 0.489 & 0.350 & 0.282 & 0.374 & 0.682 & 0.677 & 0.820 & 0.726 \\
\hline DPL-45 & 13.97 & 11.70 & 9.00 & 11.56 & 0.469 & 0.325 & 0.244 & 0.346 & 0.760 & 0.786 & 0.882 & 0.810 \\
\hline NIAB-112 & 10.37 & 7.90 & 5.50 & 7.92 & 0.289 & 0.219 & 0.189 & 0.232 & 0.597 & 0.738 & 0.764 & 0.700 \\
\hline CIM-608 & 8.17 & 6.10 & 4.27 & 6.18 & 0.295 & 0.221 & 0.163 & 0.226 & 0.498 & 0.519 & 0.615 & 0.544 \\
\hline IUB-2011 & 8.70 & 6.70 & 4.00 & 6.47 & 0.367 & 0.236 & 0.218 & 0.274 & 0.557 & 0.691 & 0.586 & 0.611 \\
\hline PB-38 & 10.27 & 7.40 & 4.87 & 7.51 & 0.443 & 0.268 & 0.217 & 0.309 & 0.613 & 0.714 & 0.669 & 0.665 \\
\hline CIM-534 & 9.60 & 6.10 & 5.10 & 6.93 & 0.318 & 0.248 & 0.190 & 0.252 & 0.586 & 0.528 & 0.680 & 0.598 \\
\hline CIM-612 & 10.13 & 6.37 & 4.30 & 6.93 & 0.439 & 0.264 & 0.253 & 0.319 & 0.649 & 0.601 & 0.580 & 0.610 \\
\hline CIM-473 & 10.40 & 6.70 & 4.20 & 7.1 & 0.418 & 0.302 & 0.228 & 0.316 & 0.672 & 0.679 & 0.577 & 0.643 \\
\hline L-229-29-71 & 9.50 & 7.47 & 5.93 & 7.63 & 0.416 & 0.337 & 0.211 & 0.322 & 0.625 & 0.728 & 0.886 & 0.746 \\
\hline B-452 & 7.80 & 6.00 & 4.40 & 6.07 & 0.468 & 0.318 & 0.250 & 0.346 & 0.487 & 0.489 & 0.564 & 0.513 \\
\hline Stone ville- 603 & 8.57 & 5.93 & 3.43 & 5.98 & 0.404 & 0.301 & 0.220 & 0.308 & 0.456 & 0.459 & 0.392 & 0.436 \\
\hline Tree Cotton & 13.40 & 11.40 & 9.20 & 11.33 & 0.536 & 0.347 & 0.299 & 0.394 & 0.727 & 0.764 & 0.800 & 0.764 \\
\hline BP-52 & 9.67 & 6.70 & 4.80 & 7.06 & 0.465 & 0.307 & 0.289 & 0.353 & 0.684 & 0.705 & 0.663 & 0.684 \\
\hline Cooker-312 & 9.87 & 7.47 & 5.37 & 7.57 & 0.337 & 0.255 & 0.195 & 0.262 & 0.599 & 0.719 & 0.661 & 0.660 \\
\hline RA-31-21 & 10.13 & 7.43 & 4.57 & 7.38 & 0.434 & 0.302 & 0.260 & 0.332 & 0.703 & 0.849 & 0.703 & 0.751 \\
\hline MS-64 & 6.77 & 5.03 & 3.17 & 4.99 & 0.364 & 0.250 & 0.177 & 0.264 & 0.561 & 0.674 & 0.781 & 0.672 \\
\hline CIM-84 & 7.43 & 5.43 & 3.93 & 5.6 & 0.294 & 0.227 & 0.205 & 0.242 & 0.424 & 0.437 & 0.472 & 0.444 \\
\hline AC-307 & 12.43 & 9.70 & 6.50 & 9.54 & 0.465 & 0.322 & 0.271 & 0.353 & 0.679 & 0.659 & 0.753 & 0.697 \\
\hline NIAB-78 & 10.10 & 6.80 & 5.90 & 7.6 & 0.351 & 0.278 & 0.228 & 0.286 & 0.650 & 0.619 & 0.820 & 0.696 \\
\hline GH-11-9-75 & 9.80 & 7.43 & 5.80 & 7.68 & 0.516 & 0.358 & 0.267 & 0.380 & 0.632 & 0.713 & 0.777 & 0.708 \\
\hline CIM-86 & 9.00 & 6.20 & 4.50 & 6.57 & 0.488 & 0.356 & 0.219 & 0.354 & 0.552 & 0.518 & 0.538 & 0.536 \\
\hline CIM-43 & 11.23 & 8.50 & 5.80 & 8.51 & 0.435 & 0.316 & 0.183 & 0.311 & 0.723 & 0.753 & 0.746 & 0.741 \\
\hline Karishma & 9.03 & 6.70 & 5.03 & 6.92 & 0.355 & 0.264 & 0.219 & 0.279 & 0.597 & 0.698 & 0.694 & 0.663 \\
\hline
\end{tabular}




\begin{tabular}{|c|c|c|c|c|c|c|c|c|c|c|c|c|}
\hline Cooker-315 & 8.10 & 5.10 & 3.07 & 5.42 & 0.300 & 0.219 & 0.152 & 0.224 & 0.538 & 0.574 & 0.615 & 0.576 \\
\hline SLH-41 & 10.77 & 6.77 & 4.87 & 7.47 & 0.454 & 0.350 & 0.266 & 0.357 & 0.648 & 0.577 & 0.569 & 0.598 \\
\hline Cyto-62 & 13.07 & 10.80 & 7.90 & 10.59 & 0.476 & 0.360 & 0.231 & 0.356 & 0.766 & 0.748 & 0.868 & 0.794 \\
\hline CRIS-134 & 9.77 & 6.17 & 4.23 & 6.72 & 0.322 & 0.283 & 0.197 & 0.268 & 0.662 & 0.642 & 0.538 & 0.614 \\
\hline CRIS-9 & 10.43 & 7.87 & 6.00 & 8.1 & 0.400 & 0.310 & 0.278 & 0.329 & 0.591 & 0.587 & 0.657 & 0.612 \\
\hline ME15 & 11.37 & 8.70 & 7.27 & 9.11 & 0.468 & 0.275 & 0.178 & 0.307 & 0.601 & 0.596 & 0.982 & 0.726 \\
\hline CIM-57 & 10.40 & 7.20 & 4.27 & 7.29 & 0.447 & 0.232 & 0.160 & 0.280 & 0.720 & 0.683 & 0.569 & 0.657 \\
\hline F-14 & 10.17 & 7.40 & 6.33 & 7.97 & 0.521 & 0.348 & 0.293 & 0.387 & 0.738 & 0.884 & 1.106 & 0.910 \\
\hline S-71 & 11.20 & 8.20 & 5.00 & 8.13 & 0.439 & 0.250 & 0.170 & 0.286 & 0.627 & 0.712 & 0.656 & 0.665 \\
\hline CIM-496 & 12.40 & 8.50 & 5.63 & 8.84 & 0.431 & 0.329 & 0.272 & 0.344 & 0.6946 & 0.642 & 0.600 & 0.645 \\
\hline
\end{tabular}

Table 3. Mean values of 50 Gossypium hirsutum $\mathbf{L}$. accessions for shoot parameters grown in control and water stress conditions

\begin{tabular}{|c|c|c|c|c|c|c|c|c|c|c|c|c|c|c|c|c|c|c|c|c|}
\hline \multirow[b]{2}{*}{ Genotypes } & \multicolumn{4}{|c|}{ SL } & \multicolumn{4}{|c|}{ SW } & \multicolumn{4}{|c|}{ DSW } & \multicolumn{4}{|c|}{ RW:SW } & \multicolumn{4}{|c|}{ DRW:DSW } \\
\hline & 100 & 70 & 40 & $\begin{array}{c}\text { M. } \\
\text { Value }\end{array}$ & 100 & 70 & 40 & $\begin{array}{c}\text { M. } \\
\text { Value }\end{array}$ & 100 & 70 & 40 & $\begin{array}{c}\text { M. } \\
\text { Value }\end{array}$ & 100 & 70 & 40 & $\begin{array}{c}\text { M. } \\
\text { Value }\end{array}$ & 100 & 70 & 40 & $\begin{array}{c}\text { M. } \\
\text { Value }\end{array}$ \\
\hline GS-444 & 12.37 & 7.47 & 4.17 & 8.00 & 1.37 & 0.85 & 0.57 & 0.93 & 0.280 & 0.151 & 0.103 & 0.178 & 0.243 & 0.238 & 0.273 & 0.251 & 0.168 & 0.211 & 0.222 & 0.201 \\
\hline Cyto-124 & 15.40 & 12.13 & 8.00 & 11.84 & 1.81 & 1.62 & 1.22 & 1.55 & 0.394 & 0.299 & 0.175 & 0.289 & 0.213 & & 0.222 & 0.202 & 0.148 & 0.148 & 0.202 & 0.166 \\
\hline DNH-105 & 17.37 & 13.40 & 10.37 & 13.71 & 2.24 & 1.73 & 1.35 & 1.77 & 0.493 & 0.320 & 0.183 & 0.332 & 0.168 & & 0.179 & 0.171 & 0.116 & 0.138 & 0.249 & 0.168 \\
\hline CRIS-533 & 18.23 & 14.37 & 10.20 & 14.27 & 1.84 & 1.43 & 1.22 & 1.50 & 0.484 & 0.314 & 0.193 & 0.330 & 0.203 & 0.183 & 0.175 & 0.187 & 0.117 & 0.134 & 0.209 & 0.154 \\
\hline MPS-27 & 15.13 & 11.53 & 8.80 & 11.82 & 1.74 & 1.26 & 1.15 & 1.38 & 0.382 & 0.225 & 0.189 & 0.265 & 0.224 & 0.228 & 0.180 & 0.211 & 0.150 & 0.191 & 0.174 & 0.172 \\
\hline CIM-506 & 14.57 & 10.80 & 8.50 & 11.29 & 1.91 & 1.45 & 0.91 & 1.42 & 0.455 & 0.298 & 0.202 & 0.318 & 0.182 & 0.190 & 0.237 & 0.203 & 0.126 & 0.140 & 0.186 & 0.151 \\
\hline TH-112/05 & 17.63 & 12.67 & 8.50 & 12.93 & 1.82 & 1.35 & 0.99 & 1.39 & 0.489 & 0.295 & 0.181 & 0.322 & 0.221 & 0.231 & 0.221 & 0.224 & 0.113 & 0.136 & 0.201 & 0.150 \\
\hline PB-896 & 18.27 & 12.37 & 8.10 & 12.91 & 1.65 & 1.13 & 0.99 & 1.26 & 0.391 & 0.214 & 0.165 & 0.257 & 0.248 & 0.266 & 0.272 & 0.262 & 0.137 & 0.195 & 0.206 & 0.179 \\
\hline Sun-02 & 19.57 & 14.63 & 9.63 & 14.61 & 2.13 & 1.39 & 0.86 & 1.46 & 0.454 & 0.306 & 0.198 & 0.319 & 0.199 & 0.230 & 0.314 & 0.248 & 0.130 & 0.154 & 0.202 & 0.162 \\
\hline CIM-573 & 17.23 & 12.67 & 7.47 & 12.46 & 1.76 & 1.43 & 1.16 & 1.45 & 0.425 & 0.291 & 0.215 & 0.310 & 0.257 & 0.206 & 0.151 & 0.205 & 0.120 & 0.141 & 0.160 & 0.140 \\
\hline BH-176 & 18.73 & 15.70 & 10.40 & 14.94 & 2.24 & 1.72 & 1.48 & 1.82 & 0.574 & 0.398 & 0.332 & 0.435 & 0.207 & 0.186 & 0.183 & 0.192 & 0.104 & 0.126 & 0.123 & 0.118 \\
\hline CIM-591 & 16.93 & 11.80 & 7.83 & 12.20 & 1.44 & 1.02 & 0.80 & 1.09 & 0.375 & 0.250 & 0.203 & 0.276 & 0.219 & 0.219 & 0.257 & 0.232 & 0.151 & 0.189 & 0.198 & 0.179 \\
\hline NIA-80 & 16.23 & 10.23 & 6.53 & 11.00 & 1.66 & 1.25 & 0.88 & 1.26 & 0.370 & 0.250 & 0.200 & 0.273 & 0.216 & 0.227 & 0.275 & 0.240 & 0.136 & 0.169 & 0.183 & 0.163 \\
\hline CRIS-510 & 16.50 & 12.27 & 8.30 & 12.36 & 1.73 & 1.39 & 0.91 & 1.34 & 0.437 & 0.306 & 0.170 & 0.304 & 0.199 & 0.187 & 0.230 & 0.205 & 0.135 & 0.148 & 0.239 & 0.174 \\
\hline VH-300 & 18.70 & 14.93 & 9.43 & 14.36 & 2.04 & 1.46 & 0.85 & 1.45 & 0.453 & 0.284 & 0.208 & 0.315 & 0.156 & 0.168 & 0.253 & 0.192 & 0.124 & 0.158 & 0.194 & 0.159 \\
\hline VS-212 & 19.67 & 15.27 & 9.73 & 14.89 & 2.11 & 1.65 & 0.99 & 1.58 & 0.472 & 0.310 & 0.240 & 0.341 & 0.132 & 0.133 & 0.171 & 0.145 & 0.115 & 0.139 & 0.168 & 0.141 \\
\hline MPS-11 & 18.90 & 15.80 & 10.50 & 15.07 & 1.75 & 1.83 & 1.28 & 1.62 & 0.562 & 0.391 & 0.328 & 0.427 & 0.279 & 0.192 & 0.220 & 0.230 & 0.105 & 0.123 & 0.134 & 0.121 \\
\hline DPL-45 & 18.37 & 14.90 & 10.20 & 14.49 & 2.24 & 1.77 & 1.27 & 1.76 & 0.562 & 0.383 & 0.338 & 0.427 & 0.209 & 0.184 & 0.192 & 0.195 & 0.113 & 0.127 & 0.133 & 0.124 \\
\hline NIAB-112 & 17.37 & 10.70 & 7.20 & 11.76 & 2.24 & 1.75 & 1.17 & 1.72 & 0.474 & 0.315 & 0.250 & 0.346 & 0.129 & 0.125 & 0.162 & 0.139 & 0.115 & 0.141 & 0.155 & 0.137 \\
\hline CIM-60 & 16.40 & 11.77 & 6.97 & 11.71 & 2.12 & 1.67 & 0.85 & 1.54 & 0.420 & 0.310 & 0.207 & 0.312 & 0.139 & 0.133 & 0.194 & 0.155 & 0.119 & 0.137 & 0.158 & 0.138 \\
\hline IUB-2011 & 15.67 & 9.70 & 6.83 & 10.73 & 1.85 & 1.23 & 0.79 & 1.29 & 0.438 & 0.320 & 0.212 & 0.323 & 0.199 & 0.192 & 0.277 & 0.223 & 0.131 & 0.146 & 0.190 & 0.155 \\
\hline
\end{tabular}


Akbar \& Hussain

\begin{tabular}{|c|c|c|c|c|c|c|c|c|c|c|c|c|c|c|c|c|c|c|c|c|}
\hline PB-38 & 16.77 & 10.37 & 7.30 & 11.48 & 1.57 & 1.15 & 0.82 & 1.18 & 0.388 & 0.206 & 0.168 & 0.254 & 0.281 & 0.233 & 0.264 & 0.260 & 0.127 & 0.195 & 0.203 & 0.175 \\
\hline CIM-534 & 16.40 & 11.60 & 7.50 & 11.83 & 2.04 & 1.35 & 0.86 & 1.42 & 0.447 & 0.233 & 0.198 & 0.293 & 0.156 & 0.183 & 0.222 & 0.187 & 0.117 & 0.169 & 0.182 & 0.156 \\
\hline CIM-612 & 15.60 & 10.60 & 7.43 & 11.21 & 1.77 & 1.14 & 0.86 & 1.25 & 0.334 & 0.251 & 0.196 & 0.260 & 0.249 & 0.232 & 0.296 & 0.259 & 0.153 & 0.155 & 0.183 & 0.163 \\
\hline CIM-473 & 15.50 & 9.87 & 7.30 & 10.89 & 1.54 & 1.07 & 0.75 & 1.12 & 0.322 & 0.260 & 0.170 & 0.251 & 0.272 & 0.283 & 0.306 & 0.287 & 0.158 & 0.154 & 0.225 & 0.179 \\
\hline L-229-29-71 & 15.20 & 10.27 & 6.70 & 10.72 & 1.34 & 0.92 & 0.63 & 0.97 & 0.347 & 0.210 & 0.175 & 0.244 & 0.310 & 0.370 & 0.334 & 0.338 & 0.159 & 0.202 & 0.189 & 0.183 \\
\hline B-452 & 16.07 & 12.27 & 7.80 & 12.04 & 1.83 & 1.70 & 0.93 & 1.49 & 0.459 & 0.292 & 0.194 & 0.315 & 0.256 & 0.187 & 0.270 & 0.238 & 0.127 & 0.159 & 0.164 & 0.150 \\
\hline Stone ville-603 & 18.80 & 12.93 & 8.77 & 13.50 & 1.93 & 1.23 & 0.62 & 1.26 & 0.444 & 0.245 & 0.190 & 0.293 & 0.209 & 0.244 & 0.358 & 0.271 & 0.127 & 0.186 & 0.226 & 0.180 \\
\hline Tree Cotton & 18.43 & 14.93 & 11.50 & 14.96 & 2.24 & 1.64 & 1.13 & 1.67 & 0.553 & 0.376 & 0.351 & 0.426 & 0.240 & 0.212 & 0.266 & 0.239 & 0.113 & 0.132 & 0.141 & 0.128 \\
\hline BP-52 & 14.13 & 9.53 & 7.23 & 10.30 & 1.66 & 1.16 & 0.84 & 1.22 & 0.387 & 0.250 & 0.204 & 0.280 & 0.280 & 0.266 & 0.343 & 0.296 & 0.144 & 0.188 & 0.214 & 0.182 \\
\hline Cooker-312 & 16.47 & 10.40 & 8.13 & 11.67 & 1.68 & 1.17 & 0.85 & 1.24 & 0.395 & 0.257 & 0.182 & 0.278 & 0.200 & 0.218 & 0.229 & 0.216 & 0.147 & 0.186 & 0.234 & 0.189 \\
\hline RA-31-21 & 14.43 & 8.77 & 6.50 & 9.90 & 1.76 & 1.22 & 0.84 & 1.28 & 0.346 & 0.250 & 0.213 & 0.269 & 0.247 & 0.249 & 0.309 & 0.268 & 0.173 & 0.191 & 0.200 & 0.188 \\
\hline MS-64 & 12.07 & 7.47 & 4.07 & 7.87 & 1.46 & 1.13 & 0.85 & 1.14 & 0.273 & 0.189 & 0.107 & 0.189 & 0.250 & 0.222 & 0.210 & 0.227 & 0.177 & 0.205 & 0.300 & 0.227 \\
\hline CIM-84 & 17.57 & 12.47 & 8.33 & 12.79 & 2.14 & 1.66 & 1.15 & 1.65 & 0.416 & 0.287 & 0.170 & 0.291 & 0.137 & 0.137 & 0.178 & 0.151 & 0.117 & 0.138 & 0.240 & 0.165 \\
\hline AC-307 & 18.30 & 14.73 & 8.63 & 13.89 & 2.26 & 1.78 & 1.23 & 1.76 & 0.460 & 0.270 & 0.195 & 0.308 & 0.206 & 0.181 & 0.221 & 0.203 & 0.126 & 0.153 & 0.193 & 0.157 \\
\hline NIAB-78 & 15.53 & 11.00 & 7.20 & 11.24 & 1.94 & 1.35 & 0.96 & 1.42 & 0.405 & 0.260 & 0.166 & 0.277 & 0.181 & 0.206 & 0.237 & 0.208 & 0.141 & 0.162 & 0.233 & 0.179 \\
\hline GH-11-9-75 & 15.50 & 10.43 & 7.50 & 11.14 & 1.74 & 1.25 & 0.96 & 1.32 & 0.374 & 0.235 & 0.193 & 0.268 & 0.296 & 0.287 & 0.280 & 0.288 & 0.152 & 0.179 & 0.186 & 0.172 \\
\hline CIM-86 & 16.30 & 11.97 & 8.37 & 12.21 & 1.94 & 1.39 & 0.85 & 1.39 & 0.445 & 0.281 & 0.211 & 0.312 & 0.252 & 0.257 & 0.258 & 0.256 & 0.132 & 0.164 & 0.194 & 0.163 \\
\hline CIM-43 & 15.53 & 11.30 & 7.80 & 11.54 & 1.78 & 1.34 & 1.18 & 1.43 & 0.348 & 0.290 & 0.193 & 0.277 & 0.245 & 0.237 & 0.156 & 0.213 & 0.153 & 0.145 & 0.179 & 0.159 \\
\hline Karishma & 15.13 & 9.60 & 7.27 & 10.67 & 1.74 & 1.15 & 0.95 & 1.28 & 0.342 & 0.262 & 0.214 & 0.273 & 0.204 & 0.230 & 0.230 & 0.221 & 0.147 & 0.163 & 0.171 & 0.160 \\
\hline Cooker-315 & 15.07 & 8.90 & 5.00 & 9.66 & 1.52 & 0.92 & 0.66 & 1.03 & 0.277 & 0.190 & 0.109 & 0.192 & 0.197 & 0.239 & 0.230 & 0.222 & 0.175 & 0.205 & 0.322 & 0.234 \\
\hline SLH-41 & 16.63 & 11.73 & 8.57 & 12.31 & 1.84 & 1.35 & 0.75 & 1.31 & 0.358 & 0.325 & 0.175 & 0.286 & 0.247 & 0.259 & 0.357 & 0.288 & 0.158 & 0.135 & 0.236 & 0.177 \\
\hline Cyto-62 & 17.10 & 14.43 & 9.10 & 13.54 & 2.22 & 1.86 & 1.65 & 1.91 & 0.552 & 0.390 & 0.350 & 0.431 & 0.214 & 0.194 & 0.140 & 0.183 & 0.113 & 0.126 & 0.132 & 0.124 \\
\hline CRIS-134 & 14.77 & 9.60 & 7.87 & 10.74 & 1.62 & 1.24 & 0.84 & 1.23 & 0.405 & 0.292 & 0.206 & 0.301 & 0.199 & 0.233 & 0.235 & 0.222 & 0.126 & 0.141 & 0.190 & 0.152 \\
\hline CRIS-9 & 17.67 & 13.40 & 9.13 & 13.40 & 1.83 & 0.93 & 0.64 & 1.14 & 0.420 & 0.263 & 0.192 & 0.292 & 0.219 & 0.332 & 0.432 & 0.327 & 0.128 & 0.164 & 0.212 & 0.168 \\
\hline ME15 & 18.90 & 14.60 & 7.40 & 13.63 & 2.24 & 1.64 & 1.07 & 1.65 & 0.460 & 0.223 & 0.185 & 0.289 & 0.209 & 0.168 & 0.166 & 0.181 & 0.115 & 0.195 & 0.190 & 0.167 \\
\hline CIM-57 & 14.47 & 10.53 & 7.50 & 10.83 & 1.62 & 1.35 & 0.95 & 1.31 & 0.367 & 0.253 & 0.261 & 0.294 & 0.277 & 0.172 & 0.169 & 0.206 & 0.152 & 0.182 & 0.161 & 0.165 \\
\hline F-14 & 13.77 & 8.37 & 5.73 & 9.29 & 1.76 & 1.46 & 0.83 & 1.35 & 0.443 & 0.276 & 0.181 & 0.300 & 0.296 & 0.239 & 0.355 & 0.297 & 0.134 & 0.170 & 0.238 & 0.181 \\
\hline S-71 & 17.90 & 11.53 & 7.63 & 12.36 & 1.75 & 1.23 & 1.04 & 1.34 & 0.431 & 0.232 & 0.186 & 0.283 & 0.252 & 0.204 & 0.164 & 0.207 & 0.134 & 0.189 & 0.170 & 0.164 \\
\hline CIM-496 & 18.03 & 13.27 & 9.40 & 13.57 & 1.91 & 1.34 & 0.94 & 1.40 & 0.452 & 0.274 & 0.196 & 0.307 & 0.226 & 0.245 & 0.291 & 0.254 & 0.128 & 0.168 & 0.215 & 0.170 \\
\hline
\end{tabular}

Table 4. Analysis of variance (Mean squares) for physiological attributes

\begin{tabular}{|c|c|c|c|c|c|c|c|c|c|}
\hline \multirow[b]{2}{*}{$\begin{array}{l}\text { Source of } \\
\text { variation }\end{array}$} & \multirow{2}{*}{$\begin{array}{c}\text { Degrees } \\
\text { of } \\
\text { freedom }\end{array}$} & \multicolumn{8}{|c|}{ Mean squares } \\
\hline & & RWC & $\begin{array}{l}\text { Exceed leaf } \\
\text { water loss }\end{array}$ & Cell injury & $\begin{array}{c}\text { Leaf water } \\
\text { potential }\end{array}$ & $\begin{array}{c}\text { Stomatal } \\
\text { conductance }\end{array}$ & $\begin{array}{l}\text { Transpirati } \\
\text { on rate }\end{array}$ & $\begin{array}{c}\text { Photo- } \\
\text { synthesis }\end{array}$ & $\begin{array}{l}\text { Osmotic } \\
\text { potential }\end{array}$ \\
\hline Replication & 2 & $3.88^{\mathrm{NS}}$ & $0.00510^{\mathrm{NS}}$ & $1.11^{\mathrm{NS}}$ & 0.813 & $0.28^{\mathrm{NS}}$ & $0.0046^{\mathrm{NS}}$ & $0.043^{\mathrm{NS}}$ & 0.000012 \\
\hline Treatment (T) & 1 & $58688.05^{* *}$ & $14.24412^{* * *}$ & $35154.19^{* *}$ & $1600.830^{* *}$ & $11149.28 * *$ & $263.3345^{* *}$ & $1240.073^{* *}$ & $0.570462 * *$ \\
\hline Genotype (G) & $\begin{array}{l}49 \\
49\end{array}$ & $167.44 * *$ & $0.57059 * *$ & $202.18 * *$ & $8.637 * *$ & $19.62 * *$ & $0.6015^{* *}$ & $1.442 * *$ & $0.006871 * *$ \\
\hline T x G & $\begin{array}{r}49 \\
198\end{array}$ & $69.18 * *$ & $0.17472 * *$ & $101.46^{* *}$ & $5.442 * *$ & $10.96 * *$ & $0.1895 * *$ & $0.473^{* *}$ & $0.001818^{* *}$ \\
\hline $\begin{array}{l}\text { Error } \\
\text { Total }\end{array}$ & $\begin{array}{l}190 \\
299\end{array}$ & 5.02 & 0.00821 & 2.69 & 0.948 & 0.21 & 0.0120 & 0.028 & 0.000096 \\
\hline
\end{tabular}

NS = Non-significant $(\mathrm{P}>0.05) ; *=$ significant $(\mathrm{P}<0.05) ; * *=$ highly significant $(\mathrm{P}<0.01), \mathrm{RWC}$ : Relative water content 
Table 5. Means of Physiological parameters of 50 Gossypium hirsutum L. accessions grown in normal and drought conditions

\begin{tabular}{|c|c|c|c|c|c|c|c|c|c|c|c|c|}
\hline \multirow{2}{*}{ Genotypes } & \multicolumn{3}{|c|}{ Relative water contents } & \multicolumn{3}{|c|}{ Exceed leaf water loss } & \multicolumn{3}{|c|}{ Cell injury } & \multicolumn{3}{|c|}{ Leaf water potential } \\
\hline & Normal & Drought & Mean & Normal & Drought & Mean & Normal & Drought & Mean & Normal & Drought & Mean \\
\hline GS-444 & 63.33 & 32.33 & 47.83 & 0.53 & 0.43 & 0.48 & 38.37 & 66.07 & 52.22 & -22.00 & -27.00 & -24.50 \\
\hline Cyto-124 & 76.33 & 46.00 & 61.17 & 0.93 & 0.53 & 0.73 & 32.97 & 55.73 & 44.35 & -19.33 & -24.67 & -22.00 \\
\hline DNH-105 & 73.67 & 41.00 & 57.33 & 1.17 & 1.00 & 1.08 & 33.73 & 61.50 & 47.62 & -17.33 & -25.33 & -21.33 \\
\hline CRIS-533 & 74.33 & 43.00 & 58.67 & 0.82 & 0.57 & 0.70 & 23.47 & 65.50 & 44.48 & -22.33 & -26.33 & -24.33 \\
\hline MPS-27 & 77.00 & 47.67 & 62.33 & 0.77 & 0.53 & 0.65 & 28.80 & 55.27 & 42.03 & -20.67 & -24.67 & -22.67 \\
\hline CIM-506 & 72.67 & 48.67 & 60.67 & 1.10 & 0.70 & 0.90 & 29.13 & 56.53 & 42.83 & -19.00 & -25.33 & -22.17 \\
\hline TH-112/05 & 75.33 & 52.00 & 63.67 & 1.30 & 0.77 & 1.03 & 35.40 & 63.67 & 49.53 & -22.00 & -25.67 & -23.84 \\
\hline PB-896 & 76.33 & 45.33 & 60.83 & 0.83 & 0.50 & 0.67 & 25.77 & 58.00 & 41.88 & -22.33 & -24.00 & -23.17 \\
\hline Sun-02 & 74.33 & 46.00 & 60.17 & 1.27 & 1.00 & 1.13 & 38.87 & 56.83 & 47.85 & -20.00 & -25.33 & -22.67 \\
\hline CIM-573 & 76.33 & 48.33 & 62.33 & 1.87 & 0.83 & 1.35 & 32.87 & 61.07 & 46.97 & -18.00 & -26.00 & -22.00 \\
\hline BH-176 & 81.00 & 58.67 & 69.83 & 2.03 & 1.47 & 1.75 & 21.10 & 39.30 & 30.20 & -17.00 & -23.00 & -20.00 \\
\hline CIM-591 & 83.00 & 51.00 & 67.00 & 1.10 & 0.73 & 0.92 & 36.87 & 61.73 & 49.30 & -22.00 & -24.00 & -23.00 \\
\hline NIA-80 & 75.33 & 49.33 & 62.33 & 0.83 & 0.60 & 0.72 & 31.57 & 48.13 & 39.85 & -22.00 & -24.67 & -23.34 \\
\hline CRIS-510 & 74.67 & 34.67 & 54.67 & 0.84 & 0.60 & 0.72 & 34.97 & 49.30 & 42.13 & -22.00 & -25.00 & -23.50 \\
\hline VH-300 & 76.00 & 41.00 & 58.50 & 0.87 & 0.73 & 0.80 & 29.10 & 51.67 & 40.38 & -21.00 & -26.00 & -23.50 \\
\hline VS-212 & 76.00 & 52.00 & 64.00 & 1.10 & 0.90 & 1.00 & 25.83 & 61.43 & 43.63 & -20.67 & -25.00 & -22.84 \\
\hline MPS-11 & 80.67 & 59.33 & 70.00 & 1.93 & 1.20 & 1.57 & 25.93 & 38.07 & 32.00 & -18.00 & -23.00 & -20.50 \\
\hline DPL-45 & 80.33 & 54.33 & 67.33 & 1.87 & 1.00 & 1.43 & 21.47 & 36.63 & 29.05 & -19.33 & -22.33 & -20.83 \\
\hline NIAB-112 & 78.33 & 52.33 & 65.33 & 1.00 & 0.43 & 0.72 & 38.23 & 48.97 & 43.60 & -22.67 & -24.33 & -23.50 \\
\hline CIM-608 & 77.33 & 50.00 & 63.67 & 1.33 & 0.80 & 1.07 & 22.07 & 53.67 & 37.87 & -19.00 & -25.33 & -22.17 \\
\hline IUB-2011 & 78.00 & 41.33 & 59.67 & 0.83 & 0.67 & 0.75 & 27.53 & 55.03 & 41.28 & -20.00 & -26.00 & -23.00 \\
\hline PB-38 & 72.33 & 40.33 & 56.33 & 0.90 & 0.70 & 0.80 & 33.27 & 47.97 & 40.62 & -20.67 & -26.33 & -23.50 \\
\hline CIM-534 & 77.33 & 41.33 & 59.33 & 0.73 & 0.70 & 0.72 & 27.87 & 60.20 & 44.03 & -21.67 & -23.67 & -22.67 \\
\hline CIM-612 & 76.00 & 48.33 & 62.17 & 1.37 & 0.63 & 1.00 & 30.13 & 49.13 & 39.63 & -22.33 & -23.33 & -22.83 \\
\hline CIM-473 & 77.00 & 53.00 & 65.00 & 0.83 & 0.50 & 0.67 & 29.17 & 54.87 & 42.02 & -22.67 & -23.67 & -23.17 \\
\hline L-229-29-71 & 71.67 & 48.00 & 59.83 & 0.90 & 0.67 & 0.78 & 32.47 & 50.97 & 41.72 & -22.00 & -25.00 & -23.50 \\
\hline B-452 & 73.00 & 49.67 & 61.33 & 0.57 & 0.90 & 0.73 & 32.20 & 41.07 & 36.63 & -19.00 & -26.33 & -22.67 \\
\hline Stone ville-603 & 71.67 & 47.33 & 59.50 & 0.97 & 1.10 & 1.03 & 31.03 & 52.20 & 41.62 & -17.00 & -25.33 & -21.17 \\
\hline Tree Cotton & 79.00 & 52.67 & 65.83 & 1.97 & 1.20 & 1.58 & 22.03 & 31.60 & 26.82 & -17.00 & -22.00 & -19.50 \\
\hline BP-52 & 71.33 & 48.33 & 59.83 & 0.87 & 0.67 & 0.77 & 29.80 & 47.73 & 38.77 & -19.67 & -25.67 & -22.67 \\
\hline Cooker-312 & 74.67 & 51.67 & 63.17 & 1.03 & 0.37 & 0.70 & 35.27 & 55.47 & 45.37 & -22.00 & -26.00 & -24.00 \\
\hline RA-31-21 & 72.33 & 40.67 & 56.50 & 1.30 & 0.47 & 0.88 & 26.17 & 55.20 & 40.68 & -20.00 & -24.33 & -22.17 \\
\hline MS-64 & 44.00 & 40.67 & 42.33 & 0.63 & 0.23 & 0.43 & 42.57 & 67.10 & 54.83 & -22.33 & -27.00 & -24.67 \\
\hline CIM-84 & 73.67 & 42.33 & 58.00 & 0.83 & 0.43 & 0.63 & 27.67 & 51.30 & 39.48 & -22.33 & -24.33 & -23.33 \\
\hline AC-307 & 75.33 & 42.00 & 58.67 & 0.93 & 0.53 & 0.73 & 37.03 & 45.60 & 41.32 & -22.67 & -25.00 & -23.84 \\
\hline NIAB-78 & 72.67 & 50.33 & 61.50 & 0.90 & 0.67 & 0.78 & 32.70 & 60.37 & 46.53 & -22.00 & -26.33 & -24.17 \\
\hline GH-11-9-75 & 77.67 & 44.33 & 61.00 & 1.70 & 0.53 & 1.12 & 31.60 & 60.00 & 45.80 & -19.33 & -26.33 & -22.83 \\
\hline CIM-86 & 73.67 & 40.67 & 57.17 & 0.80 & 0.47 & 0.63 & 26.07 & 42.83 & 34.45 & -22.00 & -26.67 & -24.34 \\
\hline CIM-43 & 79.00 & 41.33 & 60.17 & 0.80 & 0.53 & 0.67 & 36.60 & 41.87 & 39.23 & -21.00 & -25.33 & -23.17 \\
\hline
\end{tabular}


Akbar \& Hussain

\begin{tabular}{|c|c|c|c|c|c|c|c|c|c|c|c|c|}
\hline Karishma & 76.00 & 54.67 & 65.33 & 0.73 & 0.73 & 0.73 & 35.77 & 42.07 & 38.92 & -19.33 & -25.67 & -22.50 \\
\hline Cooker-315 & 45.33 & 40.00 & 42.67 & 0.57 & 0.33 & 0.45 & 35.40 & 65.53 & 50.47 & -22.00 & -27.67 & -24.84 \\
\hline SLH-41 & 77.67 & 46.67 & 62.17 & 1.53 & 0.80 & 1.17 & 30.10 & 56.17 & 43.13 & -18.67 & -25.00 & -21.84 \\
\hline Cyto-62 & 80.33 & 47.00 & 63.67 & 2.00 & 1.20 & 1.60 & 22.27 & 36.57 & 29.42 & -18.00 & -22.00 & -20.00 \\
\hline CRIS-134 & 79.33 & 42.33 & 60.83 & 1.63 & 0.50 & 1.07 & 34.17 & 52.60 & 43.38 & -20.00 & -25.67 & -22.84 \\
\hline CRIS-9 & 70.67 & 42.33 & 56.50 & 1.70 & 0.53 & 1.12 & 37.07 & 49.70 & 43.38 & -19.00 & -25.33 & -22.17 \\
\hline ME-115 & 73.33 & 47.00 & 60.17 & 0.93 & 0.60 & 0.77 & 27.70 & 62.67 & 45.18 & -18.33 & -26.00 & -22.17 \\
\hline CIM-57 & 78.00 & 43.33 & 60.67 & 0.70 & 0.43 & 0.57 & 30.07 & 52.17 & 41.12 & -20.67 & -26.67 & -23.67 \\
\hline F-14 & 71.67 & 45.00 & 58.33 & 0.87 & 0.47 & 0.67 & 30.07 & 51.13 & 40.60 & -21.00 & -25.33 & -23.17 \\
\hline S-71 & 74.67 & 45.33 & 60.00 & 0.87 & 0.43 & 0.65 & 31.97 & 44.20 & 38.08 & -22.00 & -24.67 & -23.34 \\
\hline CIM-496 & 74.67 & 44.67 & 59.67 & 1.77 & 0.53 & 1.15 & 22.47 & 44.83 & 33.65 & -22.00 & -24.67 & -23.34 \\
\hline
\end{tabular}

Table 6. Means of Physiological parameters of 50 Gossypium hirsutum L. accessions grown in normal and drought conditions

\begin{tabular}{|c|c|c|c|c|c|c|c|c|c|c|c|c|}
\hline \multirow{2}{*}{ Genotypes } & \multicolumn{3}{|c|}{ Osmotic Potential } & \multicolumn{3}{|c|}{ Photosynthesis rate } & \multicolumn{3}{|c|}{ Transpiration rate } & \multicolumn{3}{|c|}{ Stomatal Conductance } \\
\hline & Normal & Drought & Mean & Normal & Drought & Mean & Normal & Drought & Mean & Normal & Drought & Mean \\
\hline GS-444 & -0.254 & -0.350 & 0.302 & 15.05 & 11.10 & 13.08 & 4.04 & 2.18 & 3.11 & 31.45 & 21.63 & 26.54 \\
\hline Cyto-124 & -0.209 & -0.331 & 0.270 & 15.34 & 11.62 & 13.48 & 4.45 & 2.70 & 3.58 & 36.34 & 23.58 & 29.96 \\
\hline DNH-105 & -0.186 & -0.287 & 0.236 & 15.15 & 11.23 & 13.19 & 4.81 & 2.86 & 3.84 & 39.22 & 23.01 & 31.12 \\
\hline CRIS-533 & -0.187 & -0.333 & 0.260 & 15.48 & 11.35 & 13.42 & 4.29 & 2.92 & 3.61 & 34.40 & 24.25 & 29.33 \\
\hline MPS-27 & -0.171 & -0.283 & 0.227 & 15.21 & 11.98 & 13.60 & 4.35 & 3.06 & 3.70 & 32.28 & 25.06 & 28.67 \\
\hline CIM-506 & -0.205 & -0.342 & 0.273 & 15.24 & 11.42 & 13.33 & 4.92 & 2.38 & 3.65 & 36.40 & 22.62 & 29.51 \\
\hline TH-112/05 & -0.205 & -0.268 & 0.237 & 15.19 & 11.35 & 13.27 & 4.21 & 2.77 & 3.49 & 37.34 & 23.66 & 30.50 \\
\hline PB-896 & -0.198 & -0.277 & 0.237 & 15.34 & 11.39 & 13.37 & 4.95 & 2.87 & 3.91 & 34.41 & 22.37 & 28.39 \\
\hline Sun-02 & -0.192 & -0.269 & 0.231 & 15.14 & 11.45 & 13.30 & 4.52 & 2.33 & 3.43 & 39.38 & 24.78 & 32.08 \\
\hline CIM-573 & -0.170 & -0.328 & 0.249 & 15.21 & 11.40 & 13.31 & 4.98 & 2.54 & 3.76 & 34.39 & 22.76 & 28.58 \\
\hline BH-176 & -0.141 & -0.188 & 0.165 & 17.15 & 12.53 & 14.84 & 5.49 & 3.38 & 4.44 & 40.48 & 25.52 & 33.00 \\
\hline CIM-591 & -0.213 & -0.324 & 0.268 & 15.23 & 11.92 & 13.58 & 4.48 & 2.68 & 3.58 & 35.37 & 22.96 & 29.17 \\
\hline NIA-80 & -0.207 & -0.325 & 0.266 & 16.48 & 11.32 & 13.90 & 4.47 & 2.50 & 3.49 & 35.40 & 23.73 & 29.57 \\
\hline CRIS-510 & -0.206 & -0.267 & 0.237 & 15.64 & 11.46 & 13.55 & 4.94 & 3.21 & 4.08 & 36.49 & 25.00 & 30.75 \\
\hline VH-300 & -0.191 & -0.280 & 0.236 & 15.22 & 12.44 & 13.83 & 4.15 & 2.47 & 3.31 & 34.35 & 24.32 & 29.34 \\
\hline VS-212 & -0.217 & -0.260 & 0.238 & 15.85 & 11.24 & 13.55 & 4.48 & 2.97 & 3.72 & 32.10 & 24.01 & 28.06 \\
\hline MPS-11 & -0.145 & -0.254 & 0.200 & 16.98 & 13.00 & 14.99 & 5.37 & 3.32 & 4.34 & 39.84 & 25.61 & 32.73 \\
\hline DPL-45 & -0.141 & -0.254 & 0.197 & 16.59 & 13.02 & 14.81 & 5.26 & 3.41 & 4.34 & 39.60 & 25.50 & 32.55 \\
\hline NIAB-112 & -0.237 & -0.319 & 0.278 & 15.27 & 11.77 & 13.52 & 4.94 & 3.08 & 4.01 & 38.54 & 23.14 & 30.84 \\
\hline CIM-608 & -0.242 & -0.264 & 0.253 & 16.24 & 11.28 & 13.76 & 4.28 & 3.03 & 3.66 & 36.47 & 21.71 & 29.09 \\
\hline
\end{tabular}


Pure Appl. Biol., 8(1): 93-107, March, 2019

http://dx.doi.org/10.19045/bspab.2018.700167

\begin{tabular}{|c|c|c|c|c|c|c|c|c|c|c|c|c|}
\hline IUB-2011 & -0.269 & -0.263 & 0.266 & 15.13 & 11.49 & 13.31 & 4.45 & 2.76 & 3.61 & 32.20 & 22.63 & 27.42 \\
\hline PB-38 & -0.270 & -0.332 & 0.301 & 16.01 & 12.09 & 14.05 & 4.96 & 2.96 & 3.96 & 38.61 & 22.04 & 30.33 \\
\hline CIM-534 & -0.191 & -0.338 & 0.264 & 15.74 & 11.15 & 13.45 & 4.45 & 2.89 & 3.67 & 32.43 & 22.60 & 27.52 \\
\hline CIM-612 & -0.192 & -0.265 & 0.228 & 15.11 & 11.16 & 13.14 & 4.63 & 2.261 & 3.44 & 35.30 & 24.44 & 29.87 \\
\hline CIM-473 & -0.182 & -0.268 & 0.225 & 16.33 & 11.18 & 13.76 & 4.18 & 3.14 & 3.66 & 37.24 & 24.51 & 30.88 \\
\hline L-229-29-71 & -0.215 & -0.274 & 0.245 & 15.85 & 11.26 & 13.56 & 5.05 & 2.93 & 3.99 & 32.37 & 24.61 & 28.49 \\
\hline B-452 & -0.225 & -0.266 & 0.245 & 16.26 & 11.55 & 13.91 & 4.20 & 2.73 & 3.47 & 32.44 & 22.04 & 27.24 \\
\hline Stone ville- 603 & -0.190 & -0.321 & 0.256 & 15.11 & 11.22 & 13.17 & 4.43 & 2.53 & 3.48 & 32.25 & 21.84 & 27.05 \\
\hline Tree Cotton & -0.144 & -0.258 & 0.201 & 16.71 & 12.94 & 14.83 & 5.18 & 3.34 & 4.26 & 39.61 & 25.36 & 32.49 \\
\hline BP-52 & -0.183 & -0.256 & 0.220 & 15.13 & 11.47 & 13.30 & 4.30 & 2.35 & 3.33 & 33.42 & 21.90 & 27.66 \\
\hline Cooker-312 & -0.206 & -0.332 & 0.269 & 15.96 & 11.44 & 13.70 & 4.95 & 2.44 & 3.70 & 33.75 & 22.11 & 27.93 \\
\hline RA-31-21 & -0.245 & -0.351 & 0.298 & 15.22 & 11.54 & 13.38 & 4.75 & 2.51 & 3.63 & 38.47 & 22.00 & 30.24 \\
\hline MS-64 & -0.269 & -0.365 & 0.317 & 15.06 & 11.15 & 13.11 & 4.08 & 2.06 & 3.07 & 31.28 & 21.69 & 26.49 \\
\hline CIM-84 & -0.244 & -0.260 & 0.252 & 15.52 & 11.36 & 13.44 & 4.56 & 2.53 & 3.54 & 30.61 & 24.75 & 27.68 \\
\hline AC-307 & -0.252 & -0.325 & 0.288 & 16.46 & 11.23 & 13.85 & 5.08 & 2.87 & 3.98 & 35.51 & 22.17 & 28.84 \\
\hline NIAB-78 & -0.262 & -0.321 & 0.291 & 15.79 & 11.64 & 13.72 & 4.92 & 2.93 & 3.92 & 37.38 & 23.07 & 30.23 \\
\hline GH-11-9-75 & -0.245 & -0.322 & 0.284 & 15.48 & 11.34 & 13.41 & 4.20 & 2.45 & 3.33 & 32.45 & 21.79 & 27.12 \\
\hline CIM-86 & -0.215 & -0.336 & 0.276 & 15.48 & 11.80 & 13.64 & 4.55 & 2.54 & 3.55 & 35.44 & 21.95 & 28.70 \\
\hline CIM-43 & -0.237 & -0.355 & 0.296 & 15.90 & 11.18 & 13.54 & 4.68 & 2.66 & 3.67 & 38.56 & 22.83 & 30.70 \\
\hline Karishma & -0.190 & -0.263 & 0.227 & 16.02 & 12.07 & 14.05 & 4.19 & 3.05 & 3.62 & 39.45 & 24.59 & 32.02 \\
\hline Cooker-315 & -0.268 & -0.380 & 0.324 & 15.02 & 11.13 & 13.08 & 4.10 & 2.12 & 3.11 & 32.07 & 21.76 & 26.92 \\
\hline SLH-41 & -0.264 & -0.334 & 0.299 & 15.65 & 12.53 & 14.09 & 4.96 & 2.54 & 3.75 & 32.54 & 22.79 & 27.67 \\
\hline Cyto-62 & -0.153 & -0.242 & 0.198 & 17.00 & 12.96 & 14.98 & 5.10 & 3.24 & 4.17 & 39.84 & 24.91 & 32.38 \\
\hline CRIS-134 & -0.239 & -0.331 & 0.285 & 15.36 & 11.22 & 13.29 & 4.36 & 2.24 & 3.30 & 32.28 & 21.98 & 27.13 \\
\hline CRIS-9 & -0.215 & -0.263 & 0.239 & 16.16 & 12.16 & 14.16 & 4.26 & 2.58 & 3.42 & 36.49 & 24.60 & 30.55 \\
\hline ME-115 & -0.241 & -0.322 & 0.282 & 15.35 & 12.21 & 13.78 & 4.39 & 2.44 & 3.41 & 32.35 & 24.03 & 28.19 \\
\hline CIM-57 & -0.204 & -0.270 & 0.237 & 16.01 & 11.21 & 13.61 & 4.17 & 2.95 & 3.56 & 33.21 & 24.44 & 28.83 \\
\hline F-14 & -0.247 & -0.320 & 0.284 & 16.19 & 11.48 & 13.84 & 4.40 & 2.93 & 3.66 & 37.45 & 22.11 & 29.78 \\
\hline S-71 & -0.187 & -0.314 & 0.251 & 16.48 & 11.44 & 13.96 & 5.12 & 2.95 & 4.04 & 38.31 & 21.73 & 30.02 \\
\hline CIM-496 & -0.255 & -0.323 & 0.289 & 15.72 & 12.01 & 13.87 & 4.94 & 2.73 & 3.83 & 39.47 & 24.90 & 32.19 \\
\hline
\end{tabular}




\section{Conclusion}

Currently, drought stress is the main cause of severe yield losses and would be threat in future; thus, it is need of the day to develop drought-tolerant varieties on global level. We evaluated parameters that can fully differentiate between tolerant and susceptible cotton genotypes. Considerable genotypic variation found for morphological and physiological traits in response to water stress, implying that selection for drought tolerance is possible. Using our results and those of the studies cited herein, we concluded that five genotypes (BH-176, MPS-11, DPL-45, Tree cotton and Cyto-62) had high genetic potential for excellent performance under water stress condition.

\section{Authors' contributions}

Conceived and designed the experiments: $M$ Akbar, Performed the experiments: M Akbar, Analyzed the data: M Akbar \& SB Hussain, Contributed materials/ analysis/ tools: $\mathrm{M}$ Akbar \& SB Hussain, Wrote the paper: M Akbar \& SB Hussain.

\section{References}

1. United State Department of Agriculture (2015). http//:www.usda.org

2. Boyer JS (1982). Plant productivity and environment. Science (Washington, DC) 218: 443-448.

3. Boyer \& Westgate (2004). Grain yield with limited water. $J$ of Experi Bot 55: 2385-2394.

4. Turner NC (1997). Further progress in crop water relations. Adv Agron 58: 293338.

5. Sinclair TR (2005). Theoretical analysis of soil and plant traits influencing daily plant water flux on drying soils. Agron J 97: 1148-1152.

6. Alishah O \& Ahmadikhah A (2009). The effects of drought stress on improved cotton varieties in Golesatn Province of Iran. Inter J Plant Prod 3: 17-26.

7. Pace PF, Cralle HT, El-Halawany SHM, Cothren JT \& Senseman SA (1999). Drought-induced changes in shoot and root growth of young cotton plants. $J$ of Cotton Sci 3: 183-187.

8. Howard DD, Gwathney CO, Lessman GM \&Roberts RK (2001). Fertilizer additive rate and plant growth regulator effects on cotton. J Cotton Sci 5: 42-52.

9. McMichael BL \& Quisenberry JE (1991). Genetic variation for root - shoot relationship among cotton germplasm. Environ Exp Bot 31: 461-470.

10. Rahman UI, Ashraf M \& Zafar MY (2008). Genotypic variation for drought tolerance in cotton (Gossypium hirsutumL.): Gas exchange and productivity. Flora 203(2): 105-115.

11. Ullah A, Sun H, Yang X \& Zhang $X$ (2017). Drought coping strategies in cotton: increased crop per drop. Plant Biotechnol J 15(3): 271-284.

12. Pettigrew WT (2004). Physiological consequences of moisture deficit stress in cotton. Crop Sci 44: 1265-1272.

13. Sneath PHA \& Sokal RR (1973). Numerical Taxonomy: The Principles and Practice of Numerical Classification. WF Freeman \& Co., San Francisco, pp 573.

14. Maruti L \& Katageri IS (2015). Genetic influence of root traits of cotton (Gossypium hirsutum L.) on moisture stress tolerance. Karnataka J Agric Sci 28 (4): 454-458.

15. Zafar S \& Azhar M (2015). Assessment of variability for drought tolerance in Gossypium hirsutum L. at seedling stage. Pak J Agric Sci 52 (2): 301-307.

16. Iqbal K (2010). The potential for breeding upland cotton under limited water conditions. PhD. Thesis, The University of Agriculture, Faisalabad, Pakistan.

17. Bibi A, Sadaqat HA, Tahir MHN \& Akram HM (2012). Screening of sorghum (Sorghum bicolor Var. Moench) for drought tolerance at seedling stage in polyethylene glycol. J Anim Plant Sci 22(3): 671- 678.

18. Luo HH, Zhang YL \& Zhang WF (2016) Effects of water stress and re-watering on photosynthesis, root activity, and yield of 
cotton with drip irrigation under mulch. Photosynthetica 54: 65-73.

19. Hurd EA \& Spratt ED (1975). Root Pattern in Crops Related to Water and Nutrition Uptake. Physiological Aspects of Dry Land Farming (Gupta US, ed.). Oxford \& IBH Publ. Co., New Delhi.

20. Pace PF, Cralle HT, El-Halawany SHM, Cothren JT \& Senseman SA (1999). Drought-induced changes in shoot and root growth of young cotton plants. Journal of Cotton Science 3: 183-187.

21. Basal H, Bebeli P, Smith CW \& Thaxton $P$ (2003). Root growth parameters of converted race stocks of upland cotton and two $\mathrm{BC}_{2} \mathrm{~F}_{2}$ population. Crop Sci. 43: 19831988.

22. Iqbal K (2010). The potential for breeding upland cotton under limited water conditions. PhD. Thesis, The University of Agriculture, Faisalabad, Pakistan.

23. Iqbal K, Azhar FM, Khan IA \& Ullah E (2010). Assessment of cotton (Gossypium hirsutum) germplasm under water stress condition. Int J Agric Biol 12: 251-255.

24. Chaturvedi GS, Anuradha S \& Bahadur R (2012). Screening techniques for evaluating crop germplasm for drought tolerance. Plant Archives 12(1):11-18.

25. Pettigrew, W T (2004). Physiological consequences of moisture deficit stress in cotton. Crop Science 44: 1265-1272.

26. Kumar BD, Pandey M, Goswami CL \& Jain S (2001). Effect of growth regulators on photosynthesis. Transpiration and related parameters in water stressed cotton. Biol Plant 44: 475-478.

27. Fang Y \& Xiong L (2015). General mechanisms of drought response and their application in drought resistance improvement in plants Cell. Mol Life Sci 72: 673-689.

28. Nayyar H \& Gupta D (2006). Differential sensitivity of $\mathrm{C} 3$ and $\mathrm{C} 4$ plants to water deficits stress: association with oxidative stress and antioxidants. Environ and Exper Bot 58(1): 106-113.
29. Efeoglu B, Ekmekci Y \& Cicek N (2009). Physiological responses of three maize cultivars to drought stress and recovery. South African Journa 75(1): 34-42.

30. Bhutta WM (2007). The effect of cultivar on the variation of spring wheat grain quality under drought conditions. Cereal Res Commum 35: 1609-1619.

31. Ali MA, Abbas A, Niaz S \& Zulkiffal M. (2009). Morpho-physiological criteria for drought tolerance in Sorghum (Sorghum bicolor) at seedling and post-anthesis stages. Int J Agr Biol 11: 674-680.

32. Khan AI \& Sadaqat HA (2008). Heat tolerance is variable in cotton (Gossypium hirsutum L.) and can be exploited for breeding of better yielding cultivars under high temperature regimes. Pak J Bot 40: 2053-2058.

33. Azhar FM, Ali Z, Akhtar MM, Khan AA \& Trethowan R (2009). Genetic variability of heat tolerance, and its effect on yield and fibre quality traits in upland cotton (Gossypium hirsutum L.). Plant Breed 128: 356-362.

34. Chastain DR, Snider JL, Choinski JS, Collins GD, Perry CD, Whitaker J \& Grey TL (2016). Leaf ontogeny strongly influences photosynthetic tolerance to drought and high temperature in Gossypium hirsutum. J Plant Physiol 199: 18-28.

35. Deeba F, Pandey AK, Ranjan S, Mishra A, Singh R, Sharma YK \& Shirke PA (2012). Physiological and proteomic responses of cotton (Gossypium herbaceum L.) to drought stress. Plant Physiol Biochem 53: $6-18$.

36. Li D, Li C, Sun H, Liu L \& Zhang Y (2012). Photosynthetic and chlorophyll fluorescence regulation of upland cotton (Gossypium hirsutum L.) under drought conditions. Plant Omics J 5: 432- 437.

37. Chahal GS \& Gasal SS (2002). Principles and Procedures of Plant Breeding: Biotechnology and Conventional Approaches. Narosa Publishing House, New Delhi. pp 108. 